\title{
Eugenesia y anarquismo en el primer neomalthusianismo libertario barcelonés, 1896-1915
}

\author{
Eugenics and anarchism \\ in early libertarian \\ neo-Malthusianism in \\ Barcelona, 1896-1915
}

\author{
Álvaro Girón-Sierra \\ Científico titular, Grupo Cultura Médica y Científica/Institución Milá y \\ Fontanals-Consejo Superior de Investigaciones Científicas. \\ Barcelona - Catalunha - Espanha \\ agiron@imf.csic.es
}

Recebido em 30 jul. 2017.

Aprovado em 24 jan. 2018
GIRÓN-SIERRA, Álvaro. Eugenesia $\mathrm{y}$ anarquismo en el primer neomalthusianismo libertario barcelonés, 1896-1915. História, Ciências, SaúdeManguinhos, Rio de Janeiro, v.25, supl., ago. 2018, p.87-103.

Resumen

El neomalthusianismo anarquista está recibiendo una creciente atención historiográfica. El artículo pretende abordar la siguiente pregunta: ¿Tuvo algo que ver el primer neomalthusianismo anarquista barcelonés con la eugenesia? ¿Hasta qué punto y cómo? Para responder a ello el artículo tiene en cuenta el carácter transnacional del movimiento anarquista en el que las ciudades ocupaban un lugar esencial. Cosa que se materializaba en la fuerte conexión entre el grupo barcelonés y el francés, heredero de las enseñanzas de Paul Robin. La tesis del artículo es que, aunque el neomalthusianismo y el anarquismo eran movimientos distintos, el hecho de que se comparta el objetivo de regenerar la especie permitía establecer puntos de contacto aun cuando ocasionalmente los anarquistas rechazaran formalmente a la eugenesia.

Palabras clave: neomalthusianismo; eugenesia; red; degeneración; anarquismo.

Abstract

Anarchist neo-Malthusianism is increasingly receiving historiographical attention. This article deals with the following question: Did early anarchist neo-Malthusianism in Barcelona have anything to do with eugenics? To what extent, and how? To answer this, the article examines the transnational nature of the anarchist movement, in which cities played an essential role. This is shown in the strong connection between the Barcelona group and the French one, which had inherited the teachings of Paul Robin. The article's thesis is that, although neo-Malthusianism and anarchism were different movements, the fact that they shared the goal of regenerating the species allows us to establish points of contact between them, even though the anarchists formally rejected eugenics on occasion.

Keywords: neo-Malthusianism; eugenics; network; degeneration; anarchism. 


\section{Neomalthusianismo anarquista: un debate transnacional dentro del anarquismo}

El neomalthusianismo anarquista en España no es un tema poco transitado historiográficamente. El presente artículo no es un repaso de la ya importante historiografía, sino un intento por abordar el carácter de este movimiento: ¿tuvo o no tuvo que ver el neomalthusianismo anarquista con la eugenesia? Parte de la respuesta, como se verá, tiene que ver con el carácter transnacional de la discusión sobre el tema: los anarquistas de Barcelona debatían en términos que tenían mucho que ver con lo que se decía en París y Londres. Se trata de un asunto transurbano que traspasa fronteras. Tiene que ver con un tema fundamental: la obsesión generalizada, desde finales del XIX, por la degeneración de grandes masas de población en Europa. Neomalthusianismo tiene que ver con liberación sexual, pero también con mejora de la raza.

Quisiera llamar la atención sobre la dificultad del desafío. La anarquista es una subcultura. Es decir, que por mucho que hablemos de las muchas conexiones que mantuvieron con republicanos, librepensadores, y hasta cierto punto con masones, su propósito de distanciarse explícitamente de los patrones culturales de la burguesía tuvo indudables efectos (Girón Sierra, 2012). Ello para el historiador de la ciencia significa, en términos prácticos, aprender una nueva lengua, en la que términos como degeneración, eugenesia, neomalthusianismo, darwinismo, fueron resignificados ampliamente. Se puede decir todo lo que se quiera sobre el hecho indudable de que no poco del universo de lecturas de los "obreros conscientes" tenía mucho que ver con el universo de lecturas de la clase media (y eso incluía a Darwin, Spencer y Haeckel), pero también hay motivos para pensar que los mismos textos eran sometidos a procesos de apropiación y uso que desbordaban el orden cultural hegemónico (Girón Sierra, 2005, p.32). Todo ello supone, también, un desafío desde el punto de vista de la contextualización. La anarquista es una red transnacional, o más bien, un conjunto de redes informales en que muchas veces los nodos son individuos, y eso constituye un elemento a considerar (Bantman, Altena, 2017, p.9-12). Cuando se discute sobre anarquismo - o neomaltusianismo - se tienen en cuenta las condiciones locales en las que se desarrolla el debate, pero hay motivos para pensar que lo que tenía que decir al respecto Piotr Kropotkin desde Londres o Jean Grave desde París tenía gran influencia sobre sus compañeros de Barcelona. Su propio entendimiento del tiempo histórico, el alejamiento voluntario del dominio de la "política burguesa", la confianza en la ciencia combinada con la desconfianza en los científicos, crean condiciones muy especiales desde el punto de vista de la localización en el tiempo y el espacio, o si se prefiere, de la no localización en el sentido convencional del término. Los anarquistas crearon su propio mundo, no siempre fácilmente penetrable desde fuera. No es fácil, como se verá en el propio artículo, ponerse de acuerdo en una conceptualización "fuerte" de términos como eugenesia y neomalthusianismo en el caso de los ácratas.

Como es bien sabido el neomalthusianismo nace en el Reino Unido, en el entorno general del reformismo social y del socialismo utópico (Ronsin, 1979, p.87-88), aunque hay que decir que algunos de sus promotores más influyentes en el mundo anglosajón mostraban pocas simpatías con respecto a las aspiraciones del socialismo organizado, presentando el control de la natalidad más como un medio por el cual los pobres se ajustaban a las demandas de la 
economía capitalista que como un proyecto genuinamente radical (McLaren, 2000, p.170171). Sólo a partir de finales del siglo XIX, fundamentalmente en Francia, se reorientó hacia el movimiento obrero revolucionario y, ya en el XX, comenzó a penetrar en las filas del sindicalismo revolucionario (Ronsin, 1980). Y ello tuvo impacto, sobre todo, en los países del sur de Europa donde los anarquistas eran importantes. En este neomalthusianismo anarquista, el viejo amigo de Bakunin, Paul Robin, tuvo un papel fundamental, si no fundante. Robin, adherido a la Primera Internacional desde 1866 fue expulsado del Consejo de aquella al mismo tiempo que Bakunin. De hecho, su aproximación al neomalthusianismo se produce poco después de ser expulsado de la Internacional en los años 1870. Él nunca pensó que abrazar el neomalthusianismo era abandonar sus ideales revolucionarios. Por el contrario, creía que el neomalthusianismo podía llenar la falla teórica que hacía vanos todos los esfuerzos revolucionarios. Y por ello, intentó convencer a sus correligionarios anarquistas en el Congreso de Saint-Imier en 1877. Para Robin, el neomalthusianismo era un elemento estratégico imprescindible para el éxito del proletariado en la guerra de clases. Creía haber descubierto el secreto de la felicidad humana que consistía en resolver tres cuestiones: el buen nacimiento, la buena educación y la buena organización social (Drouard, 1992, p.440-441). Desgraciadamente para él, los comienzos no fueron halagüeños. En el citado congreso encontró la oposición de la plana mayor del anarquismo emergente: Kropotkin, James Guillaume, Élisée Reclus y Jean Grave. A pesar de ello, dos décadas después, en 1896, funda la Liga de la Regeneración Humana. Formada al principio por un puñado de militantes, su propaganda comenzó a tener mayores audiencias a principios del XX, a pesar de la fuerza que llegó a adquirir la propaganda natalista en Francia a las puertas de la Gran Guerra. De hecho, es el momento en el que alcanzó su apogeo allí, aupado sobre las alas de un sindicalismo revolucionario que comenzaba a tener en las filas libertarias una influencia perdurable (Ronsin, 1979, p.88-100).

¿Cuáles son los presupuestos básicos del neomalthusianismo anarquista? El presupuesto fundamental sobre el que descansaban las propuestas maltusianas era un reconocimiento explícito de la verdad de las leyes de población maltusianas, es decir, asumir la existencia de una oposición radical entre la capacidad de reproducirse de los seres organizados y el escaso alimento - y espacio - del que disponen. A pesar de los "moral checks" al aumento de la población a los que aludía Malthus, los neomalthusianos afirmaban que esto era verdad también para las poblaciones humanas. Ahora bien, en vez de proponer como el reverendo Malthus la castidad, ellos propugnaban la propaganda activa de una procreación controlada (Giroud [G. Hardy], 1904, p.4) es decir, no dejada al azar, haciendo uso de medios anticonceptivos. Desde el punto de vista de los trabajadores, el obrero no podía esperar mejorar su condición sin la limitación de nacimientos. Dicho de otra manera, había que reducir el tamaño del "ejército de reserva", o lo que es lo mismo, "elevar el precio de la carne del pueblo, provocando su escasez" (Alarcón, 1908, p.301), no solo para que suban los salarios, sino también para que en la lucha entre el capital y el trabajo los obreros estuvieran en mejores condiciones de llegar a la emancipación revolucionaria (Drouard, 1992, p.448450). Algunos pensaban, incluso, como "ante la disminución progresiva aritmética de la carne de esclavos", la emancipación no necesitaría de "sangre por las calles" (Lericolais, 1908, p.317). No era un reduccionismo del estilo del neomalthusianismo anglosajón: no 
se trataba de convertir la cuestión social en cuestión sexual. Buscaban también regenerar a los trabajadores, constituir una clase obrera sana, y, por tanto, poderosa. Su celo no se limitaba a la clase obrera, sino a la especie en su conjunto.

La oposición que encontró el neomalthusianismo en las propias filas libertarias puede ser mejor entendida si abordamos la posición al respecto del anarquista ruso Piotr Kropotkin. El elemento central de esa oposición es que no se consideraba que la contracepción fuera un elemento central en la lucha revolucionaria. Hay que considerar, además, el antimalthusianismo militante de Kropotkin y de otros como él: es el elemento clave en su reinterpretación del darwinismo (Todes, 1989, p.123-142). Para él, "la falacia de la sobrepoblación no soporta el primer intento de someterla a un examen serio" (Kropotkin, 1888 , p.831). No era un tema menor para el anarquista. En una carta de 1879 dirigida a Paul Robin, Kropoktin instaba al francés a que intentara imponer sus ideas sobre "ese maldito neomalthusianismo". Admitía que era cierto que la humanidad debiera tener en sus manos el control de su propia fertilidad - como de cualquier otra cosa - pero se rebelaba contra la idea de que la contracepción fuera un medio efectivo de prevenir la miseria. En un argumento que se repetirá hasta la saciedad, y ello incluía a una parte no pequeña de los libertarios españoles, consideraba que el neomalthusianismo no pasaba de ser otra forma de reformismo social. Hacer propaganda suya, dada su naturaleza reformista, no era muy distinto a difundir las virtudes de las cajas de ahorros o la jornada de ocho horas. Kropotkin pensaba que más valdría no perder el tiempo con paliativos, especialmente cuando de lo que se trataba era de hacer próxima la revolución (Kropotkin, 31 abr. 1879; Girón Sierra, 2010, p.133).

Había motivaciones no claramente explicitadas, pero probablemente muy poderosas. El tema del amor libre y, generalmente, el de la gestión de las pasiones, era una cuestión tratada con extraordinaria frecuencia en los medios libertarios desde la propia aparición del anarquismo, con cara y ojos. Pero ello no obsta para que hubiera no pocos libertarios que tenían una concepción bastante tradicional de las relaciones sexuales: Kropotkin no era una excepción (Woodcock, Avakumovic, 1990, p.170-171). Para el anarquista ruso, el matrimonio era demasiado sagrado como para ser profanado por curas y alcaldes (Kropotkin, 6 nov. 1882). Hay que recordar que el neomalthusianismo libertario era también un movimiento sexual especialmente avanzado. Robin, por ejemplo, afirmaba que el matrimonio monogámico y la religión eran factores de degeneración, y que el placer sexual no solo era plenamente legítimo, sino también un ingrediente esencial de una vida saludable, oponiéndose frontalmente a la continencia (McLaren, 2000, p.175). No hay divisoria entre placeres honestos y deshonestos: la causa de la prostitución se basa en la falta de respeto "universal y multiforme" a la "voluptuosidad sexual" (Robin, ago. 1907, p.172). Y fue más allá: declaró que la mujer tenía todo el derecho del mundo a disponer de su propio cuerpo sin ambages (Robin, oct. 1906, p.53).

\section{Una red española con nodo principal en Barcelona}

En cuanto a España, el neomalthusianismo anarquista, fuertemente ligado al núcleo francés liderado por Paul Robin, aparece como tal en diciembre de 1904. El grupo, encabezado por Luis Bulffi, se adhirió a la Liga de Regeneración Humana, publicando varios folletos 
y un periódico, Salud y Fuerza (1904-1914), que alcanzó una tirada más que considerable. De hecho, es la rápida implantación del neomalthusianismo anarquista lo que resulta más sorprendente. El folleto Huelga de vientres tuvo una difusión de cincuenta mil ejemplares (Bulffi, nov. 1904, p.1) cosa verdaderamente notable teniendo en cuenta las altísimas tasas de analfabetismo del país. Puede pensarse en un público de clase media barcelonés, pero Salud y Fuerza nos cuenta otra historia. Es una historia de un grupo conectado a una amplia red internacional con un nodo central francés, de un perfil ideológico más misceláneo de lo que estarían dispuestos a admitir los propios libertarios, pero que a su vez empieza a tener un gran impacto en lugares de toda la geografía española.

Los neomalthusianos anarquistas no fueron bienvenidos por todos en España, generándose un debate interno no exento de agudas aristas (Parsons, 2012). La percepción de estar doblemente perseguidos tanto por las clases dominantes, como por algunos anarquistas que lo hacían con "saña", provocaba cierta perplejidad entre los neomalthusianos (Pahissa, 29 dic. 1906, p.2.). Entre los contrarios al neomalthusianismo destacaban sonoros nombres del anarquismo catalán como Pere Esteve, Leopoldo Bonafulla o Federico Urales. Los debates internos dentro de los libertarios seguían muy de cerca lo que sucedía en Francia. En el caso de los anarquistas opuestos al neomalthusianismo, no es sorprendente que se reapropiaran de las ácidas reflexiones que hacía al respecto el grupo liderado por Jean Grave y Piotr Kropotkin en Les Temps Nouveaux. ${ }^{1}$ El argumento era parecido. El antimalthusianismo militante implicaba la negación del desequilibrio entre población y subsistencias: la miseria era el resultado del acaparamiento de la producción y la riqueza por unos pocos (Esteve [Leopoldo Bonafulla], 1905, p.6).

Los neomalthusianos anarquistas pugnaban por desactivar una crítica recurrente: el neomalthusianismo sería un lenitivo a la desastrosa situación social, nunca una solución real. O peor aún, se trataría de "una teoría de adaptación al inicuo ambiente social que nos rodea" (La prudencia..., nov. 1904, p.6). Como se decía gráficamente en El Porvenir del Obrero: "Hijo de la burguesía no muerde a su madre, sino que más bien la ayuda y la socorre" (M., 8 mar. 1907, p.3). Ciertamente, el hecho de que algunos neomalthusianos mostraran su oposición a los revolucionarios de barricada contribuía a reforzar la imagen preconstruida de contaminación burguesa dentro del campo libertario (Martínez, 1908, p.274). A ello solían responder con un hecho que consideraban constatable: si la burguesía francesa hacía todo lo posible por reprimirlo, difícilmente se puede dudar de su carácter revolucionario (Bermejo Muñoz [Aber Mein Otoonj], 23 mar. 1906, p.3.). Además, solían sostener que en ningún momento habían deslizado la idea de que la sola limitación de nacimientos fuera suficiente para resolver la "cuestión social" si se dejaban en pie religión, Estado y capital (Bulffi, feb. 1907, p.112). Por otra parte, la fe se fortificaba con las noticias del exterior: se decía que la vitalidad de la "doctrina" era tanto como que en Francia "la literatura y el teatro se habían apoderado del tema para vulgarizarlo" (Devaldés, mar. 1905, p.26). Lo cierto es que con todo lo subversivo que podía ser el neomalthusianismo anarquista en el terreno de la emancipación sexual, tenía una potencial debilidad frente a sus detractores libertarios: la Liga de Regeneración Humana era una organización internacional no compuesta exclusivamente de anarquistas. Órganos mencionados frecuentemente, como The Malthusian (Bulffi, nov. 1904, p.2), no tenían una línea editorial que les acercara precisamente a presupuestos acráticos. 
La red tenía un nodo principal en España: Barcelona. En Barcelona se estableció una biblioteca (Sección..., 1908a, p.323-324), pero también una clínica que se localizó en varios lugares de la ciudad (siguiendo, seguramente, un itinerario impulsado por los cierres gubernativos). La clínica, gratuita para los suscriptores de la revista (Por telégrafo..., 1908, p.326), se ocupaba de salud sexual, pero amplió rápidamente su campo de acción. Así, el consultorio atendía todo lo referente a las enfermedades del pulmón, corazón, y aparato digestivo; las enfermedades de la matriz (y aquí se habla de una "prestigiosa" doctora); enfermedades de la infancia; medicina general y enfermedades "secretas y urinarias" (Clínica..., dic. 1906, p.98). Se proporcionaban, también, medicamentos para "los enfermos pobres" (Sección..., 1908b, p.342). La cuestión de qué profesionales médicos estaban colaborando con los neomalthusianos, y cuál era su perfil ideológico ha sido muy poco estudiada. En todo caso, este tipo de colaboraciones interclasistas se debieron volver más difíciles cuando el anarcosindicalismo, desde los años 1910, se decantó por un perfil nítidamente obrerista que excluyó fuera del corazón del movimiento a los profesionales, intelectuales y científicos, es decir, a los no obreros (Girón Sierra, Molero Mesa, 2016).

Por otra parte, la actividad de los neomalthusianos anarquistas no pasó inadvertida para los sectores más cercanos al integrismo católico. Ello se tradujo en una serie de denuncias que solían tener, casi siempre, una resolución absolutoria en los tribunales. Lo interesante de las denuncias es que raramente se dirigían al núcleo duro ideológico de la propaganda neomalthusiana, sino a la dimensión más práctica y explícita de aquella: la difusión de los medios de prevención de la fecundación (Álvarez Peláez, 1907, p.1). La exhibición del cuerpo humano, especialmente de los órganos sexuales de mujeres y hombres en las páginas de Salud y Fuerza, se consideraba como una suerte de pornografía intolerable que ofendía a la sensibilidad de los católicos. La defensa de Luis Bulffi y su grupo, en algunos casos asumida por el republicano lerrouxista Emiliano Iglesias, ${ }^{2}$ solía ir en la línea de defender que lo único que se hacía era difundir conocimientos de higiene y salubridad sexual de manera aséptica. La "forma" importaba, y era el propio Paul Robin (oct. 1906, p.54) el que indicaba cuál era el molde adecuado de ese tipo de popularización científica: "No eran los conocimientos exactos de fisiología dados en un tiempo determinado por un profesor serio y honesto los que desmoralizan a los niños, pero sí las palabras, las canciones, las pinturas y los espectáculos de escenas bellacas y picarescas". Los neomalthusianos tenían muy claro que era fundamental la propaganda por la imagen, que era determinante para unir teoría y práctica. Expresamente se decía que en los folletos se intercalaban "infinidad de figuras anatómicas" con las cuales el propagandista puede entender mejor la materia "para explicarla después" (Generación..., 1907, p.201). Todo ello se hizo a pesar de la presión eclesiástica, aunque tuvieran que pausar en ocasiones la publicación de los artículos referentes a la higiene de los órganos sexuales, "ya que parece ser que dichos órganos no forman parte integrante del cuerpo humano, y en España es un crimen nombrarlos" (Bulffi, jul. 1907, p.166). No bastaba con afirmar la asepsia "científica" de la propaganda neomalthusiana. Había que justificar, además, de manera muy pragmática la existencia de la clínica: no se podía condenar a Bulffi y a Salud y Fuerza por luchar contra un peligro social palpable como las enfermedades venéreas. 
Hay síntomas de que la propaganda neomalthusiana tuvo cierto éxito en el hinterland de Barcelona. Y ello se muestra en la modificación de ciertos rasgos de la sociabilidad libertaria. No era inusual anunciar el registro civil de los niños, las ceremonias fúnebres no religiosas, la unión no religiosa de las parejas. Pero es significativo que, en este último caso, a una pareja de obreros de la cercana ciudad de Tarrasa se le desee "pocos tropiezos en la angustiosa vida y que si llegan a la procreación sea ésta limitada y consciente para que el fruto de sus amores pueda recibir una sana y racional educación" (Para..., 1908, p.246). Particularmente interesante, además, es el hecho de que se movilice una voluntad explícita de llegar a audiencias que trascienden un público urbano. Tal es el caso del campo andaluz. Así, se hablaba en Salud y Fuerza de dos grupos de neomalthusianos anarquistas constituidos en La Línea, uno de compañeros y otros de compañeras, teniendo el último el cometido de propagar entre las mujeres "la maternidad voluntaria". A partir de aquí se abría la perspectiva, según el órgano neomalthusiano, de que se expanda la propaganda "por toda la región andaluza" (Movimiento..., ene. 1905, p.15).

Por la propia naturaleza de las fuentes, o más bien por la inexistencia de ellas, es difícil saber hasta qué punto la propaganda neomalthusiana fue eficaz en el sur. Convendría tener en cuenta que Andalucía se convirtió en uno de los focos principales de actividad anarquista en España, además de la fuerte implantación de una sociabilidad alternativa ácrata en los pueblos andaluces. Una sociabilidad que no sólo incluía el rechazo a la política y la religión organizada, sino también un verdadero modo alternativo en el que la renuncia al consumo de bebidas alcohólicas y el vegetarianismo ocupaban un lugar no menor ¿Formaría parte del cóctel la extensión de la contracepción? Lo cierto es que en la prensa ácrata, ya en los años 1910, se empezaba a ver el neomalthusianismo, "que se ha extendido bastante, pero no lo suficiente", y la alimentación racional, que "se desconoce casi en absoluto", como elementos emancipadores complementarios (Costa Íscar, 27 mayo 1914, p.2).

Al margen de esto, Barcelona se había convertido en un nodo importante para la extensión de la red neomalthusiana en los países de lengua española. Ello permitió reivindicar al grupo de Bulffi un lugar preeminente en todo lo referente a la difusión de la buena nueva: "no hay rincón ni en España ni en la América Latina donde no figuren, entre colecciones de periódicos y libros, entre las bibliotequitas de los obreros estudiosos, sobre las mesas de lectura de Centros y Sociedades, los elegantes, económicos y, a la par, utilísimos folletos que difunde la casa editorial de Salud y Fuerza" (El problema..., 1907, p.185). Ello será especialmente verdad en el caso de Uruguay y Argentina (Masjuan Bracons, 2003, p.3034, 2017). La extensión a Buenos Aires del neomalthusiano anarquista, lugar que absorbía, fue celebrada especialmente por la publicación Salud y Fuerza (Por la..., 1908, p.356-357). ${ }^{3}$

Lo cierto es que la extensión del neomalthusianismo ácrata, incluso en zonas de poca implantación del movimiento libertario, nos hace preguntar por las propias razones del éxito: es un tema a investigar. Cabe preguntarse si la dimensión práctica de la red neomalthusiana no estaba facilitando la expansión más allá de las fronteras de los propios militantes ácratas. No solo se difunde una ideología, sino conocimientos prácticos sobre cómo evitar nacimientos indeseados. Salud y Fuerza tenía un consultorio donde se respondían las dudas de los suscriptores y lectores, que tuvo, al parecer, un éxito tan notable que lo tuvieron que limitar exclusivamente a los suscriptores de la publicación (Aviso, sep. 1907, p.187). 
La naturaleza de las preguntas y las respuestas nos hablan de consideraciones de orden bien práctico - desde posiciones presuntamente seguras en las que practicar el coito (Por telégrafo..., 1905, p.29-30) a sustancias contraceptivas propiamente dichas (Interview..., 1906, p.21-22). Además de ello, existe un terreno en el que habría que investigar con más profundidad: la propia circulación de los dispositivos. Los neomalthusianos difundieron desde Barcelona dispositivos anticonceptivos a puntos alejados de la península Ibérica: no pocos de ellos venían de Francia. La pregunta es si se trataba de un asunto puramente altruista o si había un negocio, ya que estos dispositivos no se repartían gratuitamente. ${ }^{4}$

\section{Más allá de la procreación libre de los pobres: degeneración y "antropotecnia(s)"}

¿Cuál era la naturaleza de la red neomalthusiana anarquista? ¿Tenía que ver algo el neomalthusianismo libertario con la eugenesia? Mary Nash (1984), ya en los 1980s, describía al grupo de Luis Bulffi como un grupo no muy lejos de un neomalthusianismo reformista. Eduard Masjuan Bracons (2000, p.283-288) ha sostenido la posición opuesta: lejos de una tendencia reformista, el neomalthusianismo anarquista sería un movimiento revolucionario plenamente ligado al movimiento obrero, cuyo objetivo era "la consecución del derecho de procreación libre de los pobres". Con respecto a la conexión entre neomalthusianismo y eugenesia, Masjuan Bracons declara que "el neomalthusianismo ibérico de 1900-1914 no tiene ninguna vinculación con las teorías de la eugenesia". Disociación que, desde planteamientos teóricos distintos, comparten Jorge Molero Mesa y Isabel Jiménez Lucena (2010). Cierto es que Bulffi (nov. 1904, p.2) postuló que uno de los objetivos fundamentales de la Liga de Regeneración Humana era "conquistar a la mujer la libertad de maternidad", o, mejor aún, conseguir desconectar la sexualidad de las mujeres de la maternidad. Pero también es cierto que mientras que Masjuan Bracons es especialmente convincente a la hora de demostrar que los neomalthusianos españoles de la época se mostraron generalizadamente contrarios a la práctica de la esterilización, lo es menos a la hora de demostrar la total ausencia de conexión con la eugenesia, aunque sea en un plano conceptual. Cabe aquí recordar algo sabido, pero recordado atinadamente por Richard Cleminson (2008): el carácter plural de la eugenesia, con lo que identificarla exclusivamente con programas de esterilización puede llevar a confusión. No solo porque se manejaron distintas teorías de la herencia incluyendo aquellas que admitían la inseparabilidad de medio y herencia - sino también por la enorme plasticidad política del movimiento eugenésico. En el otro extremo de la cadena nos encontramos con autores como Alain Drouard (1992), quien afirmaba para el caso francés una conexión fuerte entre eugenesia y neomalthusianismo, tanto como para decir que el eugenismo en Francia se desarrolla a partir de un terreno previamente preparado por el neomalthusianismo. Richad Sonn (2005), siguiendo esta línea, ha defendido la conexión entre neomalthusianismo anarquista y eugenesia, en especial en el período de entreguerras también en Francia. Se ha defendido, en fin, que, en todo caso, el eugenismo anarquista sería distinto al más propiamente "académico", aunque uno y otro se hicieron más difíciles de distinguir a finales de los años 1920-1930 (Diez, 2007, p.266). ${ }^{5}$ Richard Cleminson (2000, p.148-158) alude a un primer debate sobre la cuestión de la eugenesia en los años 1912-1914 en Salud y Fuerza, en el que algunos neomalthusianos - caso de José 
Chueca - se manifestaron explícitamente contrarios ala "nueva ciencia". Sin embargo, a comienzos de los años 1920, más anarquistas estuvieron dispuestos a ver a la eugenesia como algo que, trascendiendo lo "burgués", podía ser transformada en algo útil para los intereses y necesidades de los trabajadores.

No hay lugar a dudas de que el neomalthusianismo encontraba poderosos enemigos dentro de la eugenesia, institucional, por así decirlo, como Alfred Ploetz, entre otros, quien utilizó toda su artillería en el Primer Congreso de Eugenesia (1912). Para él, la propaganda malthusiana tenía un efecto contra-selectivo evidente. Dentro de las naciones eso era así porque los que más practicaban la prudencia reproductiva eran precisamente las clases más favorecidas, es decir, las que tenían mejores "variaciones", especialmente en lo referente a los "rasgos intelectuales", mientras que los pobres la practicaban menos. Pero es que, además, afectaba el equilibrio de las razas de manera notable en detrimento de las que él consideraba superiores: ingleses, holandeses, escandinavos, y alemanes, los principales "depósitos" de lo mejor de la raza nórdica, mostraban una tasa de nacimientos preocupantemente declinante, mientras que las naciones con mezcla racial asiática polacos, rusos, húngaros - tenían una tasa de nacimientos extraordinariamente alta. El neomalthusianismo no ofrecía buenas perspectivas a la raza blanca en la lucha entre las "tres grandes razas de la humanidad". Por tanto, a la espera de que el neomalthusianismo pueda ser dirigido "eugenicamente" para que dañe lo menos posible a la raza, su propaganda había de ser combatida activamente (Ploetz, 1913, p.188-189). No todos veían la cuestión así: uno de los padres fundadores del neomalthusianismo, Charles Vickery Drysdale, compartió las mismas preocupaciones referentes a la "mejora cualitativa" de la raza humana en el mismo Congreso (Section..., 1913, p.25) que los eugenistas. ${ }^{6}$ Convendría determinar hasta qué punto, los contemporáneos percibían eugenesia y neomalthusianismo como cosas distintas, o mejor aún, cuando empezaron a verse como cosas conexas. De hecho, se está cuestionando la idea de que el amplio soporte que ganó el control de natalidad en determinados países anglosajones en el período de entreguerras estuviera primariamente conectado a la defensa de los derechos de las mujeres: el éxito de las compañas de control de natalidad se basaría en su utilidad como instrumento de progreso racial. Control de la natalidad y eugenesia eran vistos como sinónimos (Carey, 2012).

Dicho de otra forma, parte del problema historiográfico que aquí se plantea - hasta qué punto tuvo que ver el neomalthusianismo anarquista con la eugenesia - tiene relación con la vaguedad de los límites de la última ¿Debiéramos definir la eugenesia bajo un marco conceptual "fuerte"? Puede que desde un punto de vista analítico genere mayor comodidad en el historiador, pero lo cierto es que el movimiento eugenésico fue plural científica y políticamente, no siendo la izquierda para nada ajena (Diane, 1984). Pero, sobre todo, hay motivos para pensar que la eugenesia encontró en su propia ambigüedad - su propia falta de cohesión interna - una de las razones de su gran expansión y crecimiento (Krementsov, 2015). Y eso hace que la respuesta en términos de blanco y negro a la pregunta de la conexión neomalthusianismo anarquista-eugenesia diste mucho de ser satisfactoria, porque por definición es imposible proveer a la eugenesia de una definición de sólidos perfiles. El caso de José Chueca ilustra lo que decimos. En 1914 Chueca identificó la eugenesia con los intentos legales de prohibir el matrimonio a aquellos que tienen enfermedades hereditarias, así como 
la esterilización de los declarados como "degenerados" por los tribunales de justicia. En el primer caso, nos encontramos con algo que paradójicamente promovería la unión libre, y, en el segundo, con un castigo "inhumano". Pero sobre todo, la eugenesia definida en estos términos es inútil. En una línea parecida a la crítica feroz de la eugenesia que hizo Kropotkin (1912) en el Congreso de Londres de 1912, Chueca consideraba que la degeneración de la especie humana seguiría su curso aun cuando se impidiera la reproducción de degenerados. Hay que ir a las causas, establecer una radical acción ambiental: suprimir las causas de la degeneración, es decir, la miseria económica. Sin embargo, Chueca (1914, p.321-322) admite tácitamente que eugenesia y neomalthusianismo comparten un fin: la regeneración de la especie. La diferencia está en los medios. Los de la eugenesia son inútiles, coercitivos y brutales, los del neomalthusianismo, son humanitarios y basados en la persuasión. Sin embargo, no todas las "eugenesias" postulaban la esterilización de los degenerados, ni tampoco excluían la acción ambiental (especialmente aquellas que partían de una teoría de la herencia neolamarckiana), ni mucho menos la "persuasión". Si en la coerción legal y estatal encontramos una valla insalvable entre estos primeros neomalthusianos barceloneses y la eugenesia, la idea de convertir el control "racional" de la reproducción humana en un instrumento contra la marea degenerativa era un terreno de fértil de contacto. Y eso es algo que se desarrolló más plenamente en los años 1920.

En realidad, la existencia de proyectos distintos de ingeniería socio-biológica no implicaba, necesariamente, que no se puedan compartir objetivos comunes. En este sentido, hay que recordar que buena parte de los contemporáneos de estos primeros neomalthusianos (Pick, 1999) pensaban también que era una necesidad imperiosa atajar la marea degenerativa. Y esto era también verdad para los anarquistas que más se oponían al neomalthusianismo. La idea general, tanto de neomalthusianos como de sus contradictores, era que el capitalismo era la causa socio-ambiental que provocaba el declive biológico de la especie, y que la única solución adecuada a la escala del problema era la revolución social. Ahora bien, no todo era esperar pasivamente el clarín revolucionario: toda una batería de medidas higiénicas eran propuestas para atenuar el avance de la degeneración (Girón Sierra, 1999). Existía, además, preocupación sobre las deletéreas consecuencias de la reproducción humana bajo la férula de la moralidad burguesa. Feroces críticos del neomalthusianismo, como eran Soledad Gustavo y su compañero Federico Urales, veían en las restricciones legales y morales al libre desarrollo de las pasiones una de las causas de la decadencia de la raza. Gustavo (1899, p.609) afirmaba que era imposible que de una unión forzada saliera un fruto robusto y "sano", mientras que Urales veía en las formas represivas de reglamentación de la unión sexual una violación de una ley de la naturaleza (Montseny, 1896, p.96). Un enemigo declarado del neomalthusianismo, como era Joan Baptista Esteve (s.f., p.59), más conocido como Leopoldo Bonafulla, sostenía que el matrimonio resultaba "nocivo para el interés de los padres y de los hijos en tanto que, en tanto que viola le ley de la selección natural". Así, el amor libre adquiría una dimensión antropotécnica: su generalización aseguraría las condiciones afectivas de una buena reproducción. Urales, por ejemplo, pensaba que los hijos "ilegítimos", resultado de una relación espontánea, eran más "geniales" y "hermosos" (Urales, 1903, p.679). 
Existía, pues, un suelo común entre los neomalthusianos y sus más feroces opositores: la preocupación por la degeneración, la búsqueda de remedios revolucionarios, o higiénicos, que lleven a la regeneración de la raza: el hombre nuevo tendría que serlo en todas las dimensiones. La discrepancia reside en los métodos: la cuestión de quién debe reproducirse estaba fuera de la agenda de los opositores al neomalthusianismo. Ya desde la primera difusión en España de las ideas de Paul Robin (1896), a través de El Corsario de La Coruña, se sabía cuál era la posición básica del nuevo malthusianismo al respecto: se ha de evitar a toda costa la propagación masiva de estirpes de degenerados, promoviendo, a la vez, la propagación de los mejor dotados. Era "deseable ... un número suficiente de individuos sanos de cuerpo" pero lo era bastante menos "tener un gran número de hijos degenerados ... en perjuicio de la infancia de calidad mejor" (p.3-4). No había dudas, la libertad sexual era un objetivo, pero la degeneración era un gran problema. En Barcelona, Bulffi utilizaba un tono muy directo. Según él, sin el neomalthusianismo todo lo que se haga y diga contra la degeneración humana resultaba ridículo. Mientras se clamaba contra esas plagas sociales, se ocultaban, por un lado, las causas de su propalación, la reproducción de los seres infectados y, por el otro, se estimulaba "que todos esos achacosos se reproduzcan como chinches, abasteciendo así a las cárceles y los hospitales" (Bulffi, feb. 1907, p.112).

Desde el punto de vista antropotécnico, la propagación de dispositivos anticonceptivos tenía una doble virtualidad. Por un lado, se posibilitaba el equilibrio entre población y subsistencias de una manera no traumática, previniendo la miseria, y, por tanto, la perpetuación de un medio patógeno. Por el otro, permitía que aquellos que sufrían enfermedades hereditarias, una vez tomada conciencia del peligro que constituía la perpetuación de sus taras para la humanidad, pudieran evitar mediante el uso de los medios anticonceptivos la propagación del mal sin renunciar a una vida sexual relativamente saludable. Existía, además del interés genérico por el bienestar y perspectivas futuras de la humanidad, una preocupación de consumo interno poderosa: la idea era crear un sujeto revolucionario fuerte, es decir, consciente de su misión histórica. Y esa tropa de "obreros conscientes" no podía estar compuesta por degenerados: al ideal del "obrero consciente" se opone necesariamente el lumpenproletariado. La cantidad, para los neomalthusianos, tenía mucho que ver con la calidad. Se asociaba la familia obrera numerosa a la propagación y perpetuación de "seres infelices, abobados, idiotizados, tuberculosos". Es decir, llevaba necesariamente a la "generación de seres inconscientes" que "son una tremenda carga" (Herrán, 1905, p.15). Y no sólo se trataba de una carga, sino de un verdadero obstáculo: la "'procreación abundante' de los proletarios solo sirve para dar vida a seres degenerados que son los apoyos con que cuenta el Estado, el Capital y la Iglesia para mantener sus privilegios" (La prudencia..., 1905, p.16). Ahora bien, no todo era preocuparse de quién debía de reproducirse, sino de asegurarse de que las condiciones ambientales para la "crianza" de la especie estuvieran aseguradas. La comparación aquí era la zootecnia. Si la reproducción, crianza y mejora de animales domésticos exigían el establecimiento de un medio adecuado, lo mismo debiera decirse de los humanos. Sin embargo, nada en ese terreno se estaba haciendo: 
Y cosa extraña. Se estudia la manera de producir mejores frutas ... Se procura que la yegua, la coneja, la gallina, hasta la cerda reciban los cuidados ... para que mejore la especie, y sin embargo nada de esto se hace para mejorar la raza humana. Se dice al obrero: ¡procrea! Y no se le facilitan ni los medios ni para que su producto sea útil y tenga viabilidad. No se le dan los conocimientos para que las condiciones físicas sean adecuadas para una producción sana, no se le dan los medios para que pueda conservar en condiciones racionales lo que produzca. El exceso de trabajo, la escasez de alimentación, la mala calidad de la misma, la falta de higiene de talleres, fábricas, trabajos en general, alojamiento, vestuarios etc. preparan la descendencia a la desperfección [sic], a la tuberculosis, a la degeneración (García, 1914, p.394).

Pero si el ambientalismo radical no estaba ausente del discurso neomalthusiano, aún estaba más presente un gran tema del degeneracionismo desde mediados del siglo XIX: la paralización de los efectos de la selección natural. Dicho de otra manera, a la selección natural fallida, habría de suceder una selección - no al azar, sino artificial, racional y consciente - basada en la prudencia en la procreación. El elemento de planificación racional no es casual. Frente al azar de la naturaleza se situaba la razón del "obrero consciente": el obrero no debe imitar a las bestias (Alarcón, sep. 1907, p.198). Si Alice Vickery (1905, p.14) decía que una Liga Neomalthusiana ha de "ocuparse de todo lo que sea convertir en racionales, elevadas y regulares las relaciones sexuales y la producción de generaciones futuras", Lluis Bulffi (nov. 1904, p.1) seguía una línea idéntica, aunque añadiendo que la descendencia no ha de ser producto del azar, sino el fruto de la voluntad consciente de "padres sanos, vigorosos de cuerpo y cerebro". La necesidad de no crear "degenerados", en realidad, impelía a no dejar casi nada al azar. El grupo neomalthusiano de Palamós, por ejemplo, establecía qué momentos eran inadecuados para practicar el coito. Así, practicar el coito en estado de embriaguez teóricamente propiciaba que se tenga una descendencia de "tuberculosos, sifilíticos, idiotas", mientras que hacerlo cuando se está sometido a un estado de decaimiento físico, lógico correlato de la miseria y las privaciones, llevaba a la propagación de "escrofulosos, anémicos y degenerados de toda clase" (Movimiento..., nov. 1905, p.29).

\section{Consideraciones finales}

Si vamos a la cuestión de un objetivo eugenésico - la mejora de la raza a través de una intervención en la reproducción humana - los neomalthusianos anarquistas difícilmente podrían ser disociados taxativamente de esa galaxia vaga a la que llamamos eugenesia, aun cuando ellos mismos se declaren, cosa del citado más arriba José Chueca, en contra de la eugenesia como tal. Pero si vamos a una intervención directa o indirecta del estado en el campo de la reproducción humana, es lógico pensar que los anarquistas poco tienen que ver en ello: los neomalthusianos, de hecho, pensaban que el estado era impotente para atajar el pauperismo y sus consecuencias en formas de miseria fisiológica (Bulffi, 1908, p.282). Algo parecido se puede decir de la ambigüedad con respecto a lo que llamamos eugenesia negativa: se solían descartar los medios traumáticos (como la esterilización), pero se tenía muy claro que había que evitar a toda costa, no por la fuerza, pero sí por la persuasión, 
que tuberculosos o sifilíticos se reprodujeran, lo cual no distinguía a los neomalthusianos barceloneses de sus compañeros franceses (Sonn, 2010, p.24). Nos encontramos con la paradoja de que se podía sostener elementos clave del credo eugenésico - la necesidad de regenerar la especie a través del control reproductivo, la admisión de que había individuos que no debían reproducirse - mientras que se rechazaba formalmente "la eugenesia" al identificarla con las esterilizaciones, la intervención del Estado, o la endeblez de una ciencia burguesa impotente antes las dimensiones de la marea degenerativa. Parte de la respuesta a esta paradoja es la aludida plasticidad del movimiento eugenésico. Pero, sobre todo, es bueno no caer en el anacronismo: Paul Robin empieza a propagar su credo neomalthusiano antes de que la eugenesia estuviera institucionalizada y fuera popular. Es cierto que eugenistas y neomalthusianos compartían un suelo común, la preocupación por la mejora cualitativa de la especie, pero eran en origen cosas distintas, aunque luego se desarrollaran concomitancias ${ }^{7}$ que no pueden ser ignoradas.

Ciertamente el rechazo a la actuación del Estado constituye una brecha importante entre buena parte del movimiento eugenésico y el neomalthusianismo anarquista. Pero la ausencia de Estado no elude formas más sutiles de poder. Responder a la pregunta de cómo evitar la propagación masiva de degenerados, implica entrar en un tema central en el anarquismo: cómo organizar una sociedad sin Estado, que hiciera posible el máximo de libertad. La ley, aparte de atentar contra la libertad, era inútil frente al deseo: "la ley no impediría dormir en una cama a tales desgraciados." La respuesta se solía encontrar en la sustitución del gobierno y la ley por el poder real de la comunidad de censurar la conducta de los individuos en pos del bien del colectivo. "¿Por qué no llevarles a la convicción de que su deber es no generalizar el mal?" (García, 1912, p.2). El problema central de los neomalthusianos anarquistas es hasta dónde se llega con esa "persuasión" a la hora de intentar impedir que determinados individuos no se reproduzcan. Un texto del propio Lluis Bulffi (1906, p.18) nos permite entender a qué nos estamos refiriendo: "Procrear familia numerosa sin poder alimentarla y huir emigrado, es una cobardía. Procrear familia numerosa sin medios de darle pan condenándola a la miseria es criminal. El que ama la vida y la libertad no procrea en la esclavitud". La estigmatización de la procreación no consciente lleva al extremo de afirmar que ésta debería reducirse a cero entre los trabajadores. Y ello porque la "procreación bestial y desenfrenada resulta criminal bajo dos aspectos, "fisiológicamente" porque se "procrean seres que hacen degenerar la especie con sus deformidades de constitución y mentalidad" $\mathrm{y}$, económicamente, porque se generan instrumentos auxiliares "de los detentadores y verdugos de la clase explotada" (Alarcón, ago. 1907, p.174).

La "persuasión", en fin, tenía el objeto de que se subjetivizara la nueva norma. Emerge una nueva moral alternativa, que se quiere alejada de la moral burguesa, pero que también pretende distanciarse de los "vicios" del proletariado. Es un tema que, sobre todo, afecta a las mujeres cuando se trata la "profilaxis" de la maternidad. Tras la información aséptica se escondían, en algunas ocasiones, exigencias que los enunciadores del discurso neomalthusiano - generalmente hombres - demandaban a las obreras conscientes: "Es cuestión primordial la educación sexual y se ha de exigir de la mujer razonable un conocimiento lo más completo posible de los órganos de la generación y su funcionamiento". Y eso cuando esos mismos hombres no estigmatizabanla propia maternidad "imprudente": 
"la mujer ha de ser aleccionada sobre la profilaxis de la maternidad para que tenga un cuidado minucioso y un interés de conciencia en evitar tan grave y desastrosa calamidad" (Costa Íscar, 8 abr. 1914, p.1.). Las afirmaciones de Costa Íscar seguramente no eran compartidas por muchos anarquistas, pero sí permite pensar que el neomalthusianismo anarquista se exponía a no pocas tensiones. Cierto es que el neomalthusianismo incluía un proyecto subversivo de radical de emancipación de las mujeres en el terreno sexual - que, por cierto, raramente incluía a la homosexualidad (Heckert, Cleminson, 2011, p.15). Pero la igualmente vehemente defensa de la prudencia reproductiva orientada a la regeneración racial, planteaba la espinosa cuestión de hasta dónde se podía llevar la "coacción moral".

\section{AGRADECIMIENTO}

El presente artículo se ha elaborado en el marco del proyecto financiado por Ministerio de Economía, Industria y Competitividad (Mineco/España): "Ciencia y ciudad. Historia natural, biología y biopolítica en la urbe dividida: Barcelona frente a Buenos Aires (1869-1936)", de referencia HAR2013-48065-C2-1P.

\section{NOTAS}

${ }^{1}$ Salud y Fuerza, por ejemplo, se ve obligado a replicar un artículo publicado por Jean Grave en Les Temps Nouveaux, luego publicado en La Revista Blanca con un título que no deja lugar a dudas: "La Sociedad Burguesa y sus 'neo' defensores" (La Sociedad..., 1904, p.4).

${ }^{2}$ Emiliano Iglesias también defendió al doctor simpatizante del anarquismo, Jaume Queralto (Calbet i Camarasa, 1999, p.136).

${ }^{3}$ En Salud y Fuerza se indicaba dónde se podía adquirir la propaganda neomalthusiana anarquista en Buenos Aires (Hacia..., jul. 1907, p.168).

${ }^{4}$ Los "conos preservativos" del doctor Mascaux se vendían al precio de 2.50 pesetas el paquete de 12 en pleno centro de Barcelona (Conos..., 1906, p.34).

${ }^{5}$ Xavier Díaz entiende que es en los años 1920-1930 cuando se detecta dentro del anarquismo español un eugenismo radical que resulta difícil de diferenciar del académico. Sin embargo, Paul Robin se mostró partidario desde temprana fecha de la esterilización (McLaren, 2000, p.177) y son incontables las veces en las que se habla de "degenerados" en la primera época de Salud y Fuerza.

${ }^{6}$ En realidad, Drysdale formó parte de la Eugenics Education Society hasta 1915 (Baker, 2014, p.291).

${ }^{7}$ En opinión de Raquel Álvarez Peláez (1995), en todo caso adquirieron más desarrollo en los años 1920-1930.

${ }^{8}$ Es el caso del importante teórico anarquista Ricardo Mella (Girón Sierra, 1994).

\section{REFERENCIAS}

ALARCÓN, José.

Generación inconsciente. Salud y Fuerza, n.23, p.301-302. 1908.

ALARCÓN, José.

Generación inconsciente. Salud y Fuerza, n.16, p.197-198. sep. 1907.

ALARCÓN, José.

Generación de esclavos. Salud y Fuerza, n.15, p.173-174. ago. 1907.

ÁLVAREZ PELÁEZ, Raquel.

Eugenesia y darwinismo social en el pensamiento anarquista. In: Hofmann,
Bert et al. (Ed.). El anarquismo español y sus tradiciones culturales. Madrid: Vervuert. p.29-40. 1995.

ÁLVAREZ PELÁEZ, Raquel.

Al lector. Salud y Fuerza, n.13, p.1. 1907.

AVISO.

Aviso. Salud y Fuerza, n.16, p.187. sep. 1907.

BAKER, Graham J.

Christianity and eugenics: the place of religion in the British Eugenics Education Society and the American Eugenics Society, c.1907-1940. Social History of Medicine, v.27, n.2, p.281-302. 2014. 
BANTAM, Constance; ALTENA, Bert. Introduction: problematizing scales of analysis in network-based social movements. In: Bantman, Constance; Altena, Bert (Ed.). Reassesing the transnational turn: scales of analysis in syndicalist studies. Oakland: PM Press. p.3-22. 2017.

\section{BERMEJO MUÑOZ, Antonio [Aber Mein} Otoonj].

La anarquía y el neomalthusianismo. El Porvenir del Obrero, n.245, p.3-4. 23 mar. 1906.

BULFFI, Lluis.

El proceso de salud y fuerza. Salud y Fuerza, n.22, p.281-283. 1908.

BULFFI, Lluis.

Medios preventivos de la fecundación. Saludy

Fuerza, n.14, p.167-168. jul. 1907.

BULFFI, Lluis.

¡Aclaración! Salud y Fuerza, n.10, p.111-114. feb. 1907.

BULFFI, Lluis.

Por el bienestar inmediato. El Nuevo Malthusiano, n.3, p.17-18. 1906.

BULFFI, Lluis.

Dos palabras. Salud y Fuerza, n.1, p.1-2. nov. 1904.

CALBET I CAMARASA, Josep Maria.

L'associació general de metges de llengua

catalana. Gimbernat, n.31, p.133-148. 1999.

CAREY, Jane.

The racial imperatives of sex: birth control and eugenics in Britain, the United States and Australia in the interwar years. Women's History Review, v.21, n.5, p.733-752. 2012.

CHUECA, José.

Eugenesia y neomalthusianismo. Salud y Fuerza, n.57, p.321-322. 1914.

CLEMINSON, Richard.

Eugenics without the State: anarchism in Catalonia, 1900-1937. Studies in History and Philosophy of Biological and Biomedical Sciences, v.39, n.2, p.232-239. 2008.

CLEMINSON, Richard.

Anarchism, science and sex: eugenics in Eastern Spain, 1900-1937. Berna: Peter Lang. 2000.

\section{CLÍNICA...}

Clínica de salud y fuerza. Salud y Fuerza, n.8, p.98. dic. 1906.

\section{CONOS..}

Conos preservativos. Salud y Fuerza, n.5, p.34. 1906.

COSTA ÍSCAR, Mariano.

Algo sobre higiene. Tierra y Libertad, n.215, p.2. 27 mayo 1914.
COSTA ÍSCAR, Mariano.

Hacia el amor libre, 4: educación sexual y procreación consciente. Tierra y Libertad, n.208, p.1. 8 abr. 1914 .

DEVALDÉS, Manuel.

Malthusianismo y neomalthusianismo. Salud y Fuerza, n.4, p.25-26. mar. 1905.

DIANE, Paul.

Eugenics and the Left. Journal of the History of Ideas, v.45, n.4, p.567-590. 1984.

DIEZ, Xavier.

El anarquismo individualista en España. Barcelona: Virus. 2007.

DROUARD, Alain.

Aux origines de l'éugénisme en France: le néomalthusianisme, 1896-1914. Population, v.47, n.2, p.435-459. 1992.

EL PROBLEMA...

El problema de la población. Salud y Fuerza, n.16, p.185. 1907.

ESTEVE, Joan Batista [Leopoldo Bonafulla].

La familia libre. Barcelona: Biblioteca Germinal. s.f.

ESTEVE, Joan Batista [Leopoldo Bonafulla].

Generación libre: los errores del

neomalthusianismo. Barcelona: Hidalgo. 1905.

GARCÍA, Vicente.

Neomalthusianismo. Salud y Fuerza, n.61, p.264265. 1914.

GARCÍA, Vicente.

Razonemos. El Porvenir del Obrero, n.314, p.2-3. 1912.

GENERACIÓN..

Generación consciente. Salud y Fuerza, n.17, p.201. 1907.

GIRÓN SIERRA, Álvaro.

Una historia contada de otra manera:

librepensamiento y "darwinismos" anarquistas en Barcelona, 1869-1910. In: Lida, Clara E.; Yankelevich, Pablo (Comp.). Cultura y política del anarquismo en España e Iberoamérica. México: Colegio de México. p.95-143. 2012.

GIRÓN SIERRA, Álvaro.

Piotr Kropotkin contra la eugenesia: siete intensos minutos. In: Miranda, Marisa; Vallejo, Gustavo (Dir.). Darwinismo social y eugenesia: derivas de Darwin: cultura y política en clave biológica. Buenos Aires: Siglo XXI; Iberoamericana. p.119-142. 2010.

GIRÓN SIERRA, Álvaro.

En la mesa con Darwin: evolución y revolución en el movimiento libertario en España. Madrid: CSIC. 2005. 
GIRÓN SIERRA, Álvaro.

Metáforas finiseculares del declive biológico: degeneración y revolución en el anarquismo español, 1872-1914. Asclepio, v.51, n.1, p.247273. 1999.

GIRÓN SIERRA, Álvaro.

Anarquismo y evolucionismo: Ricardo Mella, la coacción del grupo social y la creación sociobiológica del hombre nuevo. Asclepio, n.46, p.131-149. 1994.

GIRÓN SIERRA, Álvaro; MOLERO MESA, Jorge. The rose of fire: anarchist culture, urban spaces and management of scientific knowledge in a divided city. In: Hochadel, Oliver; Nieto-Galan, Agustí (Ed.) Barcelona: an urban history of science and modernity, 1888-1929. Nueva York: Routledge. p.115-135. 2016.

GIROUD, Gabriel [G. Hardy].

La lucha por la existencia y el

neomalthusianismo. Salud y Fuerza, v.1, n.1, p.24. nov. 1904.

GUSTAVO, Soledad.

La sociedad futura. La Revista Blanca, n.22, p.609-611. 1899.

HACIA...

¡Hacia Occidente! Salud y Fuerza, n.14, p.168. jul. 1907.

HECKERT, Jamie; CLEMINSON, Richard.

Ethics, relationships and power: an

introduction. In: Heckert, Jamie; Cleminson,

Richard (Ed.). Anarchism and sexuality: ethics, relationships and power. Nueva York: Routledge. p.1-22. 2011.

HERRÁN, J.

Al proletario. El Nuevo Malthusiano, n.2, p.14-15. 1905.

INTERVIEW...

Interview postal. El Nuevo Malthusiano, n.3, p.21-22. 1906.

KREMENTSOV, Nicolai.

The strength of a loosely defined movement: eugenics and medicine in Imperial Russia. Medical History, v.59, n.1, p.6-31. 2015.

KROPOTKIN, Piotr.

The sterilization of the unfit. Mother Earth, v.7, n.10, p.354-357. 1912.

KROPOTKIN, Piotr.

The coming reign of plenty. The Nineteenth Century, v.10, n.4, p.817-837. 1888.

KROPOTKIN, Piotr.

Carta a John Scott Keltie. Letters of Piotr Kropotkin (Royal Geographical Society/ Institute of British Geographers Archives, London). 6 nov. 1882.
KROPOTKIN, Piotr.

Carta a Paul Robin. Max Nettlau Papers, n.2702 (Internationaal Instituut voor Sociale Geschiedenis, Amsterdam). 31 abr. 1879.

LA PRUDENCIA...

La prudencia sexual y los juicios de la prensa. Salud y Fuerza, n.2, p.16. 1905.

LA PRUDENCIA...

La prudencia sexual y los juicios de la prensa. Salud y Fuerza, n.1, p.6-7. nov. 1904.

LA SOCIEDAD...

La sociedad burguesa y sus "neo" detractores. Salud y Fuerza, n.1, p.4-5. 1904.

LERICOLAIS, Eugène.

¿Para qué tener hijos? Salud y Fuerza, n.24, p.316-318. 1908.

M.

Neo-malthusianismo. El Porvenir del Obrero, n.294, p.2-3. 8 mar. 1907.

MARTÍNEZ, Miguel.

Falsas ideas de la revolución. Salud y Fuerza, n.29, p.273-276. 1908.

MASJUAN BRACONS, Eduard.

Emigración y neomalthusianismo: el ejemplo ibérico en América Latina. Historia Actual Online, n.1, p.27-37. 2003.

MASJUAN BRACONS, Eduard.

La ecología humana en el anarquismo ibérico: urbanismo "orgánico" o ecológico, neomalthusianismo y naturismo social. Barcelona: Icaria. 2000.

MCLAREN, Angus.

Reproduction and revolution: Paul Robin and neomalthusianism in France. In: Doland, Brian (Ed.). Malthus, medicine and morality: "Malthusianism" after 1798. Atlanta: Rodopi. p.264-286. 2000.

MOLERO MESA, Jorge; JIMÉNEZ LUCENA, Isabel.

"Otra manera de ver las cosas": microbios, eugenesia y ambientalismo radical en el anarquismo español del siglo XX. In: Miranda, Marisa; Vallejo, Gustavo (Dir.). Darwinismo social y eugenesia: derivas de Darwin: cultura y política en clave biológica. Buenos Aires: Siglo XXI; Iberoamericana, p.143-164. 2010.

MONTSENY, Joan.

Sociología anarquista. La Coruña: Biblioteca de "El Corsario". 1896.

MOVIMIENTO...

Movimiento emancipador. Salud y Fuerza, n.4, p.28-29. nov. 1905. 
MOVIMIENTO...

Movimiento emancipador. Salud y Fuerza, n.2, p.14-16. ene. 1905.

NASH, Mary.

El neomalthusianimo anarquista y los conocimientos populares sobre el control de la natalidad en España. In: Nash, Mary (Ed.). Presencia y protagonismo: aspectos de la historia de la mujer. Barcelona: Ediciones del Serbal. p.307-340. 1984.

PAHISSA, Lorenzo.

Neo-malthusianismo y anarquismo. El Porvenir del Obrero, n.233, p.2. 29 dic. 1906.

PARA...

Para ejemplo. Salud y Fuerza, n.19, p.246. 1908.

PARSONS, Daniel.

Neo-malthusianism, anarchism and resistance: world view and the limits of acceptance in Barcelona, 1904-1914. Entremons, n.4, p.1-18. 2012.

PICK, Daniel.

Faces of degeneration: a European disorder, c.1848-c.1918. Cambridge: Cambridge University Press. 1989.

PLOETZ, Alfred.

Neo-malthusianism and race hygiene. In: International Eugenic Congress, 1., 1912, London. Report of Proceedings, v.2. London: The Eugenics Education Society. p.183-189. 1913.

POR LA...

Por la procreación consciente. Salud y Fuerza, n.26, p.356-357. 1908.

POR TELÉGRAFO...

Por telégrafo sin hilos. Salud y Fuerza, n.24, p.326-327. 1908.

POR TELÉGRAFO...

Por telégrafo sin hilos. Salud y Fuerza, n.4, p.2930. 1905.

ROBIN, Paul.

El único remedio real de la prostitución. Salud y Fuerza, n.15, p.172-173. ago. 1907.

ROBIN, Paul.

Pan, holgura y amor. Salud y Fuerza, n.6, p.5254. oct. 1906.
ROBIN, Paul.

Regeneración: Liga para el mejoramiento de la raza humana. El Corsario, n.228, p.3-4. 1896.

RONSIN, Francis.

La grève des ventres: propagande malthusienne et baisse de la natalité en France, XIXe-XXe siècles. París: Aubier. 1980.

RONSIN, Francis.

La clase ouvrière et le néo-malthusianisme:

l'exemple français avant 1914. Le Mouvement Social, n.106, p.85-117. 1979.

SECCIÓN...

Sección de biblioteca. Salud y Fuerza, n.24, p.323-325. 1908a.

SECCIÓN...

Sección de clínica. Salud y Fuerza, n.25, p.342343. 1908b.

SECTION...

Section II: Practical eugenics. Friday, July 26th. Morning session. In: International Eugenic Congress, 1., 1912, London. Report of Proceedings, v.2. London: The Eugenics Education Society. p.22-34. 1913.

SONN, Richard.

Sex, violence, and the avant-garde: anarchism in interwar France. University Park: Pennsylvania State University Press. 2010.

SONN, Richard.

"Your body is yours": Anarchism, birth control, and eugenics in interwar France. Journal of the History of Sexuality, v.14, n.4, p.415-432. 2005.

TODES, Daniel. P.

Darwin without Malthus: the struggle for existence in Russian evolutionary thought. Oxford: Oxford University Press. 1989.

URALES, Federico.

Anarquismo: crítica de la sociedad presente: el exterminio por la vida. La Revista Blanca, n.118, p.678-681. 1903.

VICKERY, Alicia.

Liga malthusiana internacional de mujeres. El Nuevo Malthusiano, n.2, p.12-14. 1905.

WOODCOCK, George; AVAKUMOVIC, Ivan. Peter Kropotkin: from prince to rebel. Montreal: Black Rose. 1990.

\section{$\rightarrow \rightarrow \rightarrow<<<$}

